\title{
Hypergeometric functions of three variables in terms of integral representations
}

\author{
Showkat Ahmad. \\ Department of Mathematics, Sopore College, Kashmir India_193201
}

\begin{abstract}
Complete triple Hypergeometric functions of the second order which were denoted by $H_{A_{1}}, H_{B_{1}}, H_{C_{1}}$. Each of these triple Hypergeometric functions has been investigated extensively in many different ways including for example in the problem of finding their integral representations of one kind or other. Here in this paper we aim at presenting further integral representations for each triple Hypergeometric functions $H_{A_{1}}, H_{B_{1}}, H_{C_{1}}$.

Keywords: Beta and Gamma functions, Picard's integral formula, Generalized Hypergeometric functions, Gauss Hypergeometric functions, triple Hypergeometric functions, Exton's Hypergeometric functions, Appell functions.
\end{abstract}

\section{Introduction}

The great success of the theory of Hypergeometric functions of a single variable has stimulated the development of a corresponding theory in two and more variables. In $1880 \mathrm{p}$. Appell considered the product of two Gauss functions viz.

$$
{ }_{2} F_{1[]}(a, b ; c ; x)_{2} F_{1}\left(a^{\prime}, b^{\prime} ; c^{\prime} ; y\right)=\sum_{m, n=0}^{\infty} \frac{(a)_{m}\left(a^{\prime}\right)_{n}(b)_{m}\left(b^{\prime}\right)_{n}}{(c)_{m}\left(c^{\prime}\right)_{n}} \frac{x^{m}}{m !} \frac{y^{n}}{n !}
$$

We are led to five distinct possibilities of getting new functions .One such possibility is

$$
\sum_{m, n=0}^{\infty} \frac{(a)_{m+n}(b)_{m+n}}{(c)_{m+n}} \frac{x^{m}}{m !} \frac{y^{n}}{n !} .
$$

Thus the reaming four possibility lead to the four Appell function of two variables

Which are defined as $F_{1}, F_{2}, F_{3}, F_{4}$ Lauricella (1893) further generalized the four Appell functions $F_{1}, \ldots, F_{4}$ to functions of $n$ variables and defined as $F_{A}^{n}, F_{B}^{n}, F_{C}^{n}, F_{D}^{n}$ at $n=2$ that is

$$
F_{1}=F_{D}^{2}, F_{2}=F_{A}^{2}, F_{3}=F_{B}^{2} \text { and } F_{4}=F_{C}^{2}
$$

Lauricella (1893) (10, p.114) introduced 14 complete hypergeometric functions of three variables and of second order, he denoted his triple hypergeometric functions by the symbols $F_{1}, F_{2}, \ldots, F_{14}$ of which $F_{1}, F_{2}, F_{5}$, and $F_{9}$ corresponding, respectively to the three variables Lauricella functions $F_{A}^{(3)}, \quad F_{B}^{(3)}, \quad F_{C}^{(3)}, \quad F_{D}^{(3)}$ defined in [10,p.113]; see also [13, p.33,ct seq.] and [2]. Saran (1954) initiated a systematic study of ten of the triple hypergeometric function from lauricella's set. Exton [9] introduced 20 distinct triple hypergeometric functions, which he denoted by $X_{1}, \ldots, X_{20}$. Out of these Exton [9] defined and gave integral representations of some hypergeometric functions of three variables which is denoted by $X_{2}, X_{4}, X_{7}, X_{8}$, and $X_{12}$. In the course of further investigations of Lauricella complete 14 hypergeometric functions in three variables, Srivastavas $[4,5](1964 \mathrm{a}, 1967 \mathrm{~b})$ noticed the existence of three additional complete triple hypergeometric functions of the second order. These three functions are $H_{A_{1}}, H_{B_{1}}, H_{C_{1}}$ (had not been included in Lauricella set) which are defined as follows see in [11, p.43 eq. 1.5 (11) to 1.5(13)]

(1.1) $H_{A_{1}}\left(a_{1}, a_{2}, a_{3} ; c_{1}, c_{2} ; x, y, z\right)$

$$
=\sum_{m, n, p=0}^{\infty} \frac{\left(a_{1}\right)_{\mathrm{m}+\mathrm{p}}\left(a_{2}\right)_{\mathrm{m}+\mathrm{n}}\left(a_{3}\right)_{\mathrm{n}+\mathrm{p}}}{\left(\mathrm{c}_{1}\right)_{\mathrm{m}}\left(\mathrm{c}_{2}\right)_{\mathrm{n}+\mathrm{p}}} \frac{x^{m}}{m !} \cdot \frac{y^{n}}{n !} \cdot \frac{z^{p}}{p !},
$$

$(|x|=: t<1 ;|y|=: s<1 ;|z|=: t<(1-t)(1-s))$;

(1.2) $H_{B_{1}}\left(a_{1}, a_{2}, a_{3} ; c_{1}, c_{2}, c_{3} ; x, y, z\right)$

$$
=\sum_{m, n, p=0}^{\infty} \frac{\left(a_{1}\right)_{\mathrm{m}+\mathrm{p}}\left(a_{2}\right)_{\mathrm{m}+\mathrm{n}}\left(a_{3}\right)_{\mathrm{n}+\mathrm{p}}}{\left(\mathrm{c}_{1}\right)_{\mathrm{m}}\left(\mathrm{c}_{2}\right)_{\mathrm{n}}\left(\mathrm{c}_{3}\right)_{\mathrm{p}}} \frac{x^{m}}{m !} \cdot \frac{y^{n}}{n !} \cdot \frac{z^{p}}{p !}
$$

$(\mathrm{t}:=|x| ; \mathrm{s}:=|y| ; \mathrm{t}:=|z| ; t+s+t+2 \sqrt{t s t}<1)$;

(1.3) $H_{C_{1}}\left(a_{1}, a_{2}, a_{3} ; c ; x, y, z\right)$ 


$$
\begin{gathered}
=\sum_{m, n, p=0}^{\infty} \frac{\left(a_{1}\right)_{\mathrm{m}+\mathrm{p}}\left(a_{2}\right)_{\mathrm{m}+\mathrm{n}}\left(a_{3}\right)_{\mathrm{n}+\mathrm{p}}}{(\mathrm{c})_{\mathrm{m}+\mathrm{n}+\mathrm{p}}} \frac{x^{m}}{m !} \cdot \frac{y^{n}}{n !} \cdot \frac{z^{p}}{p !}, \\
(\mathrm{t}:=|x| ; \mathrm{s}:=|y| ; \mathrm{t}:=|z| ; t+s+t-2 \sqrt{(1-t)(1-s)(1-t)<2}) .
\end{gathered}
$$

Where with $\mathbb{C}$ and $\mathbb{Z}_{0}^{-}$denoting the set of complex numbers and the set of non -positive integers respectively $(\lambda) n$ is the Pochhammmer symbol defined (for $\lambda \epsilon c$ )

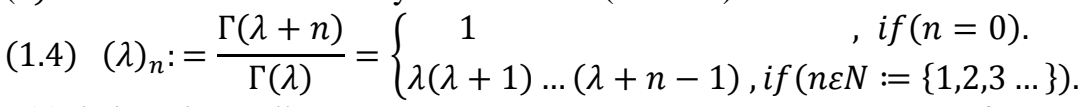

$\Gamma(\mathrm{z})$ being the well known Gamma function .Of course, all 20 of Exton's triple hypergeometric function $x_{1}, \ldots, x_{20}$ as well as Srivastava's triple hypergeometric $H_{A_{1}}, H_{B_{1}}, H_{C_{1}}$ are included in the set of the aforementioned 205 distinct triple hypergeometric function which we presented systematically by Srivastava's Karlsson [11,chapter 3]. The above stated three dimensional regions of convergence of the triple hypergeometric series (1.1), (1.2) and (1.3) for $H_{A_{1}}, H_{C_{1}}$ respt. Were given by Srivastava [4, 5] (see also Srivastava and Karlsson [11, section 3.4]).

(1.5) $H_{A_{1}}\left(\alpha, \beta_{1}, \beta_{2} ; \gamma_{1}, \gamma_{2} ; x, y, z\right)=\frac{\Gamma\left(\gamma_{1}\right) \Gamma\left(\gamma_{2}\right)}{\Gamma\left(\gamma_{1}\right) \Gamma\left(\gamma_{2}\right) \Gamma\left(\gamma_{1}-\beta_{1}\right) \Gamma\left(\gamma_{2}-\beta_{2}\right)}$

$$
\begin{aligned}
& \cdot \int_{0}^{1} \int_{0}^{1} \xi^{\beta_{1}-1} n^{\beta_{2}-1}(1-\xi)^{\gamma_{1}-\beta_{1}-1}(1-n)^{\gamma_{2}-\beta_{2}-1}(1-y n)^{-\beta_{1}}(1-x \xi-z n)^{-\alpha} \\
& \cdot\left(1-\frac{x y \xi \eta}{(1-y \eta)(1-x \xi-z \eta)}\right) d \xi d \eta,
\end{aligned}
$$

$\left(\Re\left(\gamma_{1}\right)>\Re\left(\beta_{1}\right)>0 ;\left(\Re\left(\gamma_{2}\right)>\Re\left(\beta_{2}\right)>0\right)\right.$

(1.6) $H_{C_{1}}\left(\alpha, \beta_{1}, \beta_{2} ; \gamma ; x, y, z\right)$

$$
\begin{aligned}
& =\frac{\Gamma(\gamma)}{\Gamma(\alpha) \Gamma\left(\beta_{1}\right) \Gamma\left(\gamma-\alpha-\beta_{1}\right)} \\
& \cdot \int_{0}^{1} \int_{0}^{1} \xi^{\alpha-1} \eta^{\beta-1}(1-\xi)^{\gamma-\alpha-1}(1-\eta)^{\gamma-\alpha-\beta_{1}-1}(1-x \xi)^{\beta_{2}-\beta_{1}} \\
& \cdot\left(1-x \xi-y \eta-z \xi+y \xi \eta+z x \xi^{2}\right)^{-\beta_{2}} d x \xi \eta,
\end{aligned}
$$

$\left(\min \left\{\mathfrak{R}(\alpha), \mathfrak{R}\left(\beta_{1}\right), \mathfrak{R}\left(\gamma-\alpha-\beta_{1}\right)\right\}>0\right)$.

Here in this chapter, our aim is to investigate some further integral representation for each of the three Srivastavas function $H_{A_{1}}, H_{B_{1}}, H_{C_{1}}$.

\section{Integral Representation of $\boldsymbol{H}_{A_{1}}$}

THEORM 1: - Each of the following integral representation for $H_{A_{1}}$ holds true:

(2.1) $H_{A_{1}}\left(a_{1}, a_{2}, a_{3} ; c_{1}, c_{2} ; x, y, z\right)$

$$
\begin{gathered}
=\frac{\Gamma\left(c_{2}\right)}{\Gamma\left(a_{3}\right) \Gamma\left(c_{2}-a_{3}\right)} \int_{0}^{1} \xi^{a_{3}-1}(1-\xi)^{c_{2}-a_{3}-1}(1-y \xi)^{-a_{2}} \\
H_{A_{1}}\left(a_{1}, a_{2}, a_{3} ; c_{1}, c_{2} ; x, y, z\right) \\
=\frac{\Gamma\left(c_{2}\right) \Gamma(1+\lambda)^{a_{3}}}{\Gamma\left(a_{3}\right) \Gamma\left(c_{2}-a_{3}\right)} \int_{0}^{1} \xi^{a_{3}-1}(1-\xi)^{c_{2}-a_{3}-1}(1+\lambda \xi)^{a_{1}+a_{2}-c_{2}} \\
.[1+\lambda \xi-(1+\lambda) \xi y]^{-a_{2}}[1+\lambda \xi-(1+\lambda) \xi z]^{-a_{1}} \\
.{ }_{2} F_{1}\left(a_{1}, a_{2} ; c_{1} ; \frac{x(1+\lambda \xi)^{2}}{[1+\lambda \xi-(1+\lambda) \xi y][1+\lambda \xi-(1+\lambda) \xi z]}\right) d \xi, \\
\left(\mathfrak{R}\left(c_{2}\right)>\Re\left(a_{3}\right)>0 ; \lambda>-1\right) ;
\end{gathered}
$$

(2.3) $H_{A_{1}}\left(a_{1}, a_{2}, a_{3} ; c_{1}, c_{2} ; x, y, z\right)$

$$
\begin{aligned}
& =\frac{\Gamma\left(c_{2}\right)}{\Gamma\left(a_{3}\right) \Gamma\left(c_{2}-a_{3}\right)} \frac{(\beta-\gamma)^{a_{3}}(\alpha-\gamma)^{c_{2}-a_{3}}}{(\beta-\alpha)^{c_{2}-a_{1}-a_{2-1}}} \\
& \cdot \int_{\alpha}^{\beta}(\beta-\xi)^{c_{2-} a_{3}-1}(\xi-\alpha)^{a_{3}-1}(\xi-\gamma)^{a_{1}+a_{2}-c_{2}} \\
& .[(\beta-\alpha)(\xi-\gamma)-(\beta-\gamma)(\xi-\alpha) y]^{-a_{2}} \\
& .[(\beta-\alpha)(\xi-\gamma)-(\beta-\gamma)(\xi-\alpha) z]^{-a_{1}} \\
& .{ }_{2} F_{1}\left(a_{1}, a_{2} ; c_{1} ; \sigma x\right) d \xi
\end{aligned}
$$

$\left(\Re\left(c_{2}\right)>\Re\left(a_{3}\right)>0 ; \gamma<\alpha<\beta\right)$. 
Where

$$
\sigma:=\frac{(\beta-\alpha)^{2}(\xi-\gamma)^{2}}{[(\beta-\alpha)(\xi-\gamma)-(\beta-\gamma)(\xi-\alpha) y][(\beta-\alpha)(\xi-\gamma)-(\beta-\gamma)(\xi-\alpha) z]} ;
$$

$H_{A_{1}}\left(a_{1}, a_{2}, a_{3} ; c_{1}, c_{2} ; x, y, z\right)$

$$
\begin{aligned}
& =\frac{\Gamma\left(c_{2}\right)}{\Gamma\left(a_{3}\right) \Gamma\left(c_{2}-a_{3}\right)} \int_{0}^{\infty} \xi^{a_{3}-1}(1+\xi)^{a_{1}+a_{2}-c_{2}} \\
& \cdot(1+\xi-y \xi)^{-a_{2}(1+\xi-z \xi)^{-a_{1}}} \\
& { }_{2} F_{1}\left(a_{1}, a_{2} ; c_{1} ; \frac{x(\mathbf{1}+\xi)^{2}}{(\mathbf{1}+\xi-\mathrm{y} \xi)(\mathbf{1}+\xi-\mathrm{z} \xi)}\right) d \xi,
\end{aligned}
$$

(2.5) $H_{A_{1}}\left(a_{1}, a_{2}, a_{3} ; c_{1}, c_{2} ; x, y, z\right)$

$$
\left(\Re\left(c_{2}\right)>\Re\left(a_{3}\right)>0\right) ;
$$

$$
\begin{aligned}
= & \frac{2 \Gamma\left(c_{2}\right)}{\Gamma\left(a_{3}\right) \Gamma\left(c_{2}-a_{3}\right)} \int_{0}^{\frac{\pi}{2}}\left(\sin ^{2} \xi\right)^{a_{3}-\frac{1}{2}}\left(\cos ^{2} \xi\right)^{c_{2}-a_{3}-\frac{1}{2}} \\
& \cdot\left(1-\operatorname{yin}^{2} \xi\right)^{-a_{2}} \cdot\left(1-z \sin ^{2} \xi\right)^{-a_{1}} \\
& \cdot{ }_{2} F_{1}\left(a_{1}, a_{2} ; c_{1} ; \frac{x}{\left(1-y \sin ^{2} \xi\right)\left(1-\operatorname{zsin}^{2} \xi\right)}\right) d \xi,
\end{aligned}
$$

$\left(\mathfrak{R}\left(c_{2}\right)>\Re\left(a_{3}\right)>0\right)$.

Here ${ }_{2} F_{1}$ 回 denotes the well- known gauss hypergeometric function defined by (2.6) ${ }_{2} F_{1}(\mathrm{a}, \mathrm{b}, \mathrm{c} ; \mathrm{z})$

$$
\begin{aligned}
=\sum_{\mathrm{n}=0}^{\infty} \frac{(a)_{\mathrm{n}}(\mathrm{b})_{\mathrm{n}}}{(\mathrm{c})_{\mathrm{n}}} \frac{\mathrm{z}^{\mathrm{n}}}{\mathrm{n} !} ; \quad \mathrm{c} \in \mathbb{C} \backslash \mathbb{Z}_{0}^{-}:|\mathrm{z}|<1 ;|\mathrm{z}|=1(\mathrm{z} \neq-1) \\
\text { and } \Re(\mathrm{c}-a-\mathrm{b})>0 ; z=-1 \text { and } \Re(\mathrm{c}-a-\mathrm{b})>-1
\end{aligned}
$$

\section{Proof Of Theorem $\boldsymbol{H}_{A_{1}}$}

Proof: The integral representation (2.1) was derived by Srivastavas himself [4, p. 100] as an intermediate result in his demonstration of the integral representation (1.5) [4,p. 100, eq.(3.3)]. In fact Srivastavas derivation of (2.1) involved writing the triple hypergeometric series in (1.1) as a single series of the Appell function $F_{1}$ as follows:

(3.1) $H_{A_{1}}\left(a_{1}, a_{2}, a_{3} ; c_{1}, c_{2} ; x, y, z\right)$

$$
=\sum_{m=0}^{\infty} \frac{\left(a_{1}\right)_{\mathrm{m}}\left(a_{2}\right)_{\mathrm{m}}}{\left(\mathrm{c}_{1}\right)_{\mathrm{m}}} \mathrm{F}_{1}\left[a_{3}, a_{2}+\mathrm{m}, a_{1}+\mathrm{m} ; c_{2} ; \mathrm{y}, \mathrm{z}\right] \frac{x^{m}}{m !},
$$

And then applying Picard's integral formula [12, p.29, eq.(4)]

(3.2) $F_{1}\left[\alpha, \beta, \beta^{\prime} ; \gamma ; x, y\right]$

$$
\begin{array}{r}
=\frac{\Gamma(\gamma)}{\Gamma(\alpha) \Gamma(\gamma-\alpha)} \int_{0}^{1} \tau^{\alpha-1}(1-\tau)^{\gamma-\alpha-1}(1-x t)^{-\beta}(1-y t)^{\beta^{\prime}} d \tau, \\
\quad\left(\mathrm{R}(\gamma)>R(\alpha)>0 ; \gamma \in \mathbb{C} \backslash \mathbb{Z}_{0}^{-}\right) .
\end{array}
$$

To each term on the right-hand side of (3.1). The transition from (2.1) to Srivastavas

Final result (1.5) was made by appealing to the following classical result (see, for Details, [4, pp.99-100])

(3.3) ${ }_{2} \mathrm{~F}_{1}(\alpha, \beta ; \gamma ; \mathrm{z})$

$$
=\frac{\Gamma(\gamma)}{\Gamma(\alpha) \Gamma(\gamma-\alpha)} \int_{0}^{1} \tau^{\alpha-1}(1-\tau)^{\gamma-\alpha-1}(1-z t)^{-\beta} d \tau,
$$

$\left(\mathrm{R}(\gamma)>R(\alpha)>0 ; \gamma \in \mathbb{C} \backslash \mathbb{Z}_{0}^{-}\right)$.

The assertions (2.4) and (2.5) of theorem1 would follow from Srivastava's resultupon setting

$$
\begin{aligned}
& \quad \xi \rightarrow \frac{\xi}{1-\xi} \quad d \xi \rightarrow \frac{d \xi}{(1-\xi)^{2}} \quad \text { and } \quad(0,1) \rightarrow(0, \infty) \\
& \text { and } \xi \rightarrow \sin ^{2} \xi, d \xi \rightarrow 2 \sin \xi \cos \xi d \xi \quad \text { and }(0,1) \rightarrow\left(0, \frac{\pi}{2}\right) \text { respectively. }
\end{aligned}
$$

Each of the integral representations (2.1) to (2.5) can also be proved directly by expressing the series definition of the involved hypergeometric functions ${ }_{2} F_{1}$ in each integrand and changing the order of the integral sign and the summation, and finally using one or the other of the following well- known relationships between the Beta functions $\mathrm{B}(\alpha, \beta)$, the gamma function $\Gamma(z)$ and their various associated Eulerian integral (see, for example [6, p.26 and pp. 86, problem]). 


$$
\mathrm{B}(\alpha, \beta)=\left\{\begin{array}{cl}
\int_{0}^{1} t^{\alpha-1}(1-t)^{\beta-1} \mathrm{dt}, & (\min \{\Re(\alpha)>0, \Re(\beta)\}>0) ; \\
\frac{\Gamma(\alpha) \Gamma(\beta)}{\Gamma(\alpha+\beta)}, & \left(\alpha, \beta \in \mathbb{C} \backslash \mathbb{Z}_{0}^{-}\right)
\end{array}\right.
$$

$$
\mathrm{B}(\alpha, \beta)=2 \int_{0}^{\pi / 2}(\sin \theta)^{2 \alpha-1}(\cos \theta)^{2 \beta-1} d \theta=\int_{0}^{\infty} \frac{r^{\alpha-1}}{(1+r)^{\alpha+\beta}} d r \text {, }
$$

$$
(\min \{\Re(\alpha)>0, \Re(\beta)\}>0)
$$

$$
\begin{aligned}
\mathrm{B}(\alpha, \beta) & =\frac{(b-c)^{\alpha}(a-c)^{\beta}}{(b-a)^{\alpha+\beta-1}} \int_{\mathrm{a}}^{\mathrm{b}} \frac{(t-a)^{\alpha-1}(b-t)^{\beta-1}}{(t-c)^{\alpha+\beta}} \mathrm{dt} \\
& =(1+\lambda)^{\alpha} \int_{0}^{1} \frac{\mathrm{t}^{\alpha-1}(1-t)^{\beta-1}}{(1+\lambda \mathrm{t})^{\alpha+\beta}} \mathrm{dt}, \quad \lambda>-1,
\end{aligned}
$$

\section{Integral Representation of $\boldsymbol{H}_{B_{1}}$}

THEOREM 2:- Each of the following integral representation for $H_{B_{1}}$ holds true

(4.1) $H_{B_{1}}\left(a_{1}, a_{2}, a_{3} ; c_{1}, c_{2}, c_{3} ; x, y, z\right)$

(4.2) $H_{B_{1}}\left(a_{1}, a_{2}, a_{3} ; c_{1}, c_{2}, c_{3} ; x, y, z\right)$

$$
\begin{aligned}
= & \frac{\Gamma\left(a_{1}+a_{2}\right)}{\Gamma\left(a_{1}\right) \Gamma\left(a_{2}\right)} \int_{0}^{1} \xi^{a_{1}-1}(1-\xi)^{a_{2}-1} \\
& . X_{4}\left[a_{1}+a_{2}, a_{3} ; c_{1}, c_{2}, c_{3} ; x \xi(1-\xi), y(1-\xi), z \xi\right] d \xi, \\
& \left(\min \left\{\Re\left(a_{1}\right), \Re\left(a_{2}\right)\right\}>0\right) ;
\end{aligned}
$$

$$
\begin{aligned}
= & \frac{\Gamma\left(a_{1}+a_{2}\right)}{\Gamma\left(a_{1}\right) \Gamma\left(a_{2}\right)} \frac{(\beta-\gamma)^{a_{1}}(\alpha-\gamma)^{a_{2}}}{(\beta-\alpha)^{a_{1}+a_{2}-1}} \\
& \cdot \int_{\alpha}^{\beta}(\beta-\xi)^{a_{2}-1}(\xi-\alpha)^{a_{1-1}}(\xi-\gamma)^{-a_{1}-a_{2}} \\
& \quad X_{4}\left[a_{1}+a_{2}, a_{3} ; c_{1}, c_{2}, c_{3} ; \sigma_{1} x, \sigma_{2} y, \sigma_{3} z\right] d \xi, \\
& \left(\min \left\{\mathfrak{R}\left(a_{1}\right), \Re\left(a_{2}\right)\right\}>0 ; \gamma<\alpha<\beta\right) .
\end{aligned}
$$

Where

$$
\begin{aligned}
& \sigma_{1}:= \frac{(\alpha-\gamma)(\beta-\gamma)(\xi-\alpha)(\beta-\xi)}{(\beta-\alpha)^{2}(\xi-\gamma)^{2}}, \\
& \sigma_{2}:=\frac{(\alpha-\gamma)(\beta-\xi)}{(\beta-\alpha)(\xi-\gamma)}, \sigma_{3}:=\frac{(\beta-\gamma)(\xi-\alpha)}{(\beta-\alpha)(\xi-\gamma)}
\end{aligned}
$$$$
H_{B_{1}}\left(a_{1}, a_{2}, a_{3} ; c_{1}, c_{2}, c_{3} ; x, y, z\right)
$$$$
\begin{aligned}
& =\frac{2 \Gamma\left(a_{1}+a_{2}\right)}{\Gamma\left(a_{1}\right) \Gamma\left(a_{2}\right)} \int_{0}^{\frac{\pi}{2}}\left(\sin ^{2} \xi\right)^{a_{1}-\frac{1}{2}}\left(\cos ^{2} \xi\right)^{a_{2}-\frac{1}{2}} \\
& . X_{4}\left(a_{1}+a_{2}, a_{3} ; c_{1}, c_{2}, c_{3} ; \sigma_{1} x, \sigma_{2} y, \sigma_{3} z\right) d \xi
\end{aligned}
$$

Where

(4.4) $H_{B_{1}}\left(a_{1}, a_{2}, a_{3} ; c_{1}, c_{2}, c_{3} ; x, y, z\right)$

$$
\sigma_{1}:=\sin ^{2} \xi \cos ^{2} \xi, \sigma_{2}:=\cos ^{2} \xi, \sigma_{3}:=\sin ^{2} \xi
$$

$$
\begin{aligned}
= & \frac{2 \Gamma\left(a_{1}+a_{2}\right)(1+\lambda)^{a_{1}}}{\Gamma\left(a_{1}\right) \Gamma\left(a_{2}\right)} \int_{0}^{\frac{\pi}{2}} \frac{(\sin \xi)^{a_{1}-\frac{1}{2}}\left(\cos ^{2} \xi\right)^{a_{2}-\frac{1}{2}}}{\left(1+\lambda \sin ^{2} \xi\right)^{a_{1}+a_{2}}} \\
& . X_{4}\left(a_{1}+a_{2}, a_{3} ; c_{1}, c_{2}, c_{3} ; \sigma_{1} x, \sigma_{2} y, \sigma_{3} z\right) d \xi,
\end{aligned}
$$

Where

(4.5) $H_{B_{1}}\left(a_{1}, a_{2}, a_{3} ; c_{1}, c_{2}, c_{3} ; x, y, z\right)$

$$
\sigma_{1}:=\frac{(1+\lambda) \sin ^{2} \xi \cos ^{2} \xi}{\left.(1+\lambda) \sin ^{2} \xi\right)}, \sigma_{2}:=\frac{\cos ^{2} \xi}{1+\lambda \sin ^{2} \xi}, \sigma_{3}:=\frac{(1+\lambda) \sin ^{2} \xi}{1+\lambda \sin ^{2} \xi}
$$

$$
=\frac{\Gamma\left(a_{1}+a_{2}\right) \lambda^{a_{1}}}{\Gamma\left(a_{1}\right) \Gamma\left(a_{2}\right)} \int_{0}^{\frac{\pi}{2}} \frac{\left(\sin ^{2} \xi\right)^{a_{1}-\frac{1}{2}}\left(\cos ^{2} \xi\right)^{a_{2}-\frac{1}{2}}}{\left(\cos ^{2} \xi+\sin ^{2} \xi\right)^{a_{1}+a_{2}}}
$$




$$
. X_{4}\left(a_{1}+a_{2}, a_{3} ; c_{1}, c_{2}, c_{3} ; \sigma_{1} x, \sigma_{2} y, \sigma_{3} \mathrm{z}\right) d \xi,
$$

$\left(\min \left\{\Re\left(a_{1}\right), \Re\left(a_{2}\right)\right\}>0\right)$.

Where

$$
\sigma_{1}:=\frac{\lambda \sin ^{2} \xi \cos ^{2} \xi}{\left(\cos ^{2} \xi+\lambda \sin ^{2} \xi\right)}, \sigma_{2}:=\frac{\cos ^{2} \xi}{\cos ^{2} \xi+\lambda \sin ^{2} \xi}, \sigma_{3}:=\frac{\lambda \sin ^{2} \xi}{\cos ^{2} \xi+\lambda \sin ^{2} \xi} .
$$

Here $X_{4}$ denotes one of Exton's twenty hypergeometric functions defined by (See [9] and [11, p. 84, Entry $(45 \mathrm{a})])$.

\section{Proof Of Theorem $\boldsymbol{H}_{B_{1}}$}

Proof: A similar argument as in the demonstration of theorem1 will establish the results asserted by theorem 2 . Instead of the Gauss hypergeometric series in (2.6), we make use of the double hypergeometric series in (2.6) for Exton's function $X_{4}$

Alternatively, the assertion (4.1) to (4.5) of Theorem 2 can be proven directly and much more systematically by first writing the definition (1.2) in the following form

(5.1) $\boldsymbol{H}_{\boldsymbol{B}_{1}}\left(a_{1}, a_{2}, a_{3} ; c_{1}, c_{2}, c_{3} ; x, y, z\right)$

$$
\begin{aligned}
= & \sum_{m, n, p=0}^{\infty} \frac{\left(a_{1}\right)_{\mathrm{m}+\mathrm{p}}\left(a_{2}\right)_{\mathrm{m}+\mathrm{n}}\left(a_{3}\right)_{\mathrm{n}+\mathrm{p}}}{\left(\mathrm{c}_{1}\right)_{\mathrm{m}}\left(\mathrm{c}_{2}\right)_{\mathrm{n}}\left(\mathrm{c}_{3}\right)_{\mathrm{p}}} \frac{x^{m}}{m !} \cdot \frac{y^{n}}{n !} \cdot \frac{z^{p}}{p !} \\
= & \frac{\Gamma\left(a_{1}+a_{2}\right)}{\Gamma\left(a_{1}\right) \Gamma\left(a_{2}\right)} \sum_{m, n, p=0}^{\infty} \frac{\left(a_{1}+a_{2}\right)_{2 \mathrm{~m}+\mathrm{n}+\mathrm{p}}\left(a_{1}\right)_{\mathrm{n}+\mathrm{p}}}{\left(\mathrm{c}_{1}\right)_{\mathrm{m}}\left(\mathrm{c}_{2}\right)_{\mathrm{n}}\left(\mathrm{c}_{3}\right)_{\mathrm{p}}} \frac{x^{m}}{m !} \cdot \frac{y^{n}}{n !} \cdot \frac{z^{p}}{p !} \\
& . B\left(a_{1}+m+p, a_{2}+m+n\right),
\end{aligned}
$$

$\left(\min \left\{\mathfrak{R}\left(a_{1}\right), \mathfrak{R}\left(a_{2}\right),\right\}>0\right)$.

Replace the beta function $B\left(a_{1}+m+p, a_{2}+m+n\right)$ by on or other of its numerous Eulerian representations and the interpreting the resulting triple hypergeometric series by mean of the definition (2.6). In this manner, of course, we can derive a considerably large number of other integral representations for $H_{B_{1}}$ involving the triple hypergeometric function $X_{4}$ defined by (2.6).

\section{Integral Representation Of $\boldsymbol{H}_{C_{1}}$}

THEOREM 3:- Each of the following integral representation for $H_{C_{1}}$ holds true

(6.1) $H_{C_{1}}\left(a_{1}, a_{2}, a_{3} c ; x, y, z\right)$

$$
\begin{gathered}
=\frac{\Gamma(c)}{\Gamma\left(a_{1}\right) \Gamma\left(c-a_{1}\right)} \int_{0}^{1} \xi^{a_{1}-1}(1-\xi)^{c-a_{1}-1}(1-x \xi)^{-a_{2}}(1-z \xi) \\
\cdot{ }_{2} F_{1}\left(a_{2}, a_{3} ; c-a_{1} ; \frac{y(1-\xi)}{(1-x \xi)(1-z \xi)}\right) d \xi,\left(\Re(c)>\Re\left(a_{1}\right)>0\right) ; \\
=\frac{\Gamma(c)(1+\lambda)^{a_{1}}}{\Gamma\left(a_{1}\right) \Gamma\left(c-a_{1}\right)} \int_{0}^{1} \xi^{a_{1}-1}(1-\xi)^{c-a_{1}-1} \\
.(1+\lambda \xi)^{a_{2}+a_{3}-c}[1+\lambda \xi-(1+\lambda) x \xi]^{-a_{2}}[1+\lambda \xi-(1+\lambda) z \xi] \\
H_{C_{1}}\left(a_{1}, a_{2}, a_{3} ; c ; x, y, z\right) \\
\cdot{ }_{2} F_{1}\left(a_{2}, a_{3} ; c-a_{1} ; \frac{\mathrm{y}(1+\lambda \xi)(1-\xi)}{[1+\lambda \xi-(1+\lambda) x \xi][1+\lambda \xi-(1+\lambda) z \xi]}\right) d \xi, \\
H_{C_{1}}\left(a_{1}, a_{2}, a_{3} ; \mathrm{c} ; x, y, z\right) \\
=\frac{\Gamma(c)}{\Gamma\left(a_{1}\right) \Gamma\left(c-a_{1}\right)} \frac{(\beta-\gamma)^{a_{1}}(\alpha-\gamma)^{\mathrm{c}-a_{1}}}{(\beta-\alpha)^{\mathrm{c}-a_{2}-a_{3}-1}} \\
\cdot \int_{\alpha}^{\beta}(\beta-\xi)^{c-a_{1}-1}(\xi-\alpha)^{a_{1}-1}(\xi-\gamma)^{a_{2}-a_{3}-c} \\
\cdot[(\beta-\alpha)(\xi-\gamma)-(\beta-\gamma)(\xi-\alpha) x]^{-a_{2}} \\
.[(\beta-\alpha)(\xi-\gamma)-(\beta-\gamma)(\xi-\alpha) z]^{-a_{3}}
\end{gathered}
$$




$$
{ }_{.2} F_{1}\left(a_{2}, a_{3} ; \mathrm{c}-\mathrm{a}_{1} ; \sigma \mathrm{y}\right) d \xi
$$

Where

$$
\left.\Re(c)>\Re\left(a_{1}\right)>0 ; \gamma>\alpha>\beta\right) ;
$$

$$
\begin{array}{r}
\sigma:=\frac{(\beta-\alpha)(\alpha-\gamma)(\xi-\gamma)(\beta-\xi)}{[(\beta-\alpha)(\xi-\gamma)-(\beta-\gamma)(\xi-\alpha) \mathrm{x}][(\beta-\alpha)(\xi-\gamma)-(\beta-\gamma)(\xi-\alpha) \mathrm{z}]} \\
H_{C_{1}}\left(a_{1}, a_{2}, a_{3} ; \mathrm{c} ; x, y, z\right) \\
=\frac{\Gamma(c)}{\Gamma\left(a_{1}\right) \Gamma\left(c-a_{1}\right)} \int_{0}^{1} \xi^{a_{1}-1}(1+\xi)^{a_{2}+a_{3}-c}(1+\xi-\xi \mathrm{x})^{-a_{2}} \\
.(1+\xi-\xi \mathrm{z})^{-a_{3}}{ }_{2} F_{1}\left(a_{2}, a_{3} ; \mathrm{c}-\mathrm{a}_{1} ; \sigma \mathrm{y}\right) d \xi, \quad\left(\Re(c)>\Re\left(a_{1}\right)>0\right) ;
\end{array}
$$

Where

$$
\begin{aligned}
& H_{C_{1}}\left(a_{1}, a_{2}, a_{3} ; c ; x, y, z\right) \\
& \sigma:=\frac{1+\xi}{(1+\xi-\xi x)(1+\xi-\xi \mathrm{z})} \\
& =\frac{\Gamma(c)}{\Gamma\left(a_{1}\right) \Gamma\left(c-a_{1}\right)} \int_{0}^{\frac{\pi}{2}}\left(\sin ^{2} \xi\right)^{a_{1}-1 / 2}\left(\cos ^{2} \xi\right)^{a_{2}-1 / 2} \\
& .\left(1-x \sin ^{2} \xi\right)^{-a_{2}}\left(1-\operatorname{zsin}^{2} \xi\right)^{-a_{3}}{ }_{2} F_{1}\left(a_{2}, a_{3} ; c-a_{1} ; \sigma \mathrm{y}\right) d \xi \text {, }
\end{aligned}
$$

Where

$$
\left(\mathfrak{R}(c)>\Re\left(a_{1}\right)>0\right) \text {, }
$$

$$
\sigma:=\frac{\cos ^{2} \xi}{\left(1-x \sin ^{2} \xi\right)\left(1-z \sin ^{2} \xi\right)}
$$

Here ${ }_{2} F_{1}$ denotes the gauss hypergeometric function given by (2.6)

\section{Roof Of Theorem $H_{C_{1}}$}

Proof: Now proof of Theorem 3 is much akin to that of Theorem1, which we have already presented in a reasonably detailed manner, but instead of Picard's integral formula we use another form of Picard's formula as: (3.2) $F_{1}\left[\alpha, \beta, \beta^{\prime} ; \gamma ; x, \mathrm{z}\right]$

$$
\begin{array}{r}
=\frac{\Gamma(\gamma)}{\Gamma(\alpha) \Gamma(\gamma-\alpha)} \int_{0}^{1} \tau^{\alpha-1}(1-\tau)^{\gamma+\alpha+n-1}(1-x t)^{-\beta}(1-y t)^{-\beta^{\prime}} d \tau, \\
\quad\left(\mathrm{R}(\gamma)>R(\alpha)>0 ; \gamma \in \mathbb{C} \backslash \mathbb{Z}_{0}^{-}\right) .
\end{array}
$$

And other evaluation is same as Theorem1, so that we get the required solution.

\section{CONCLUSION}

Integral representation for most of the special functions of Mathematical physics and applied Mathematics has been investigated in the existing literature. Here we have presented only some illustrative integral representation for each of Srivastavas functions $H_{A_{1}}, H_{B_{1}}, H_{C_{1}}$.A variety of integral representation of $H_{A_{1}}, H_{B_{1}}$ and $H_{C_{1}}$ which may be different from those presented here, can also be provided. Furthermore, just we mentioned in connection with the single- and double- integral representation (2.1) and (1.5) for $H_{A_{1}}$, srivastava's double- integral representation (1.6) for $H_{C_{1}}$ can easily be deduced from the assertion (6.1) of theorem 3 by appealing to the classical result (3.3).

\section{References}

[1]. Carlson, B. C. (1963). Lauricella's hypergeometric function $F_{D}$. J. Math. Anal. Appl. 7, 452-470.

[2]. Hasanov, A. Srivastava, H. M and Turaev, M. (2006). Decomposition formulas for some triple hypergeometric function. J. Math. Anal. Apple. 324, 955- 969.

[3]. Saran, S. (1954). Hypergeometric functions of three variables. Ganita, 5, 71- 91; see also Corrigendum, Ganita, 7 (1956), 65.

[4]. Srivastava, H. M (1964).Hypergeometric function of three variables. Ganita 15, 97-108.

[5]. Srivastava, H. M (1967). Some integrals representing triple hypergeometric functions, Rend. Circ. Mat. Palermo (Ser. 2), 16, 99-115.

[6]. Srivastava, H.M. and Manocha, H. L. (1984). A Treatise on generating Functions. Halsted Press, John wiley and sons, New York, Chichester, Brisbane and Toronto.

[7]. Srivastava, H. M. and Karlsson, P. W. (1985). Multiple Gaussian hypergeometric series. Halsted Press (Ellis Horwood limited, Chichester, Brisbane and Toronto.

[8]. Turaev, M. (2009). Decomposition formulas for Srivastava's hypergeometric- ric function $H_{A}$ on Saran function. J. Compute. Appl. Math, 233, 842- 846. 
[9]. Exton, H. (1982). Hypergeometric function of three variables. J. Indian Acad. Math. 4, 113-119.

[10]. Lauricella, G. (1893). Sulle funzioni iper-geometriche a piu variabili.Rend. Cire. Mat. Palermo. 7, 111- 158.

[11]. Srivastava, H. M and Karlsson, P. W. (1985). Multiple Gaussian hypergeometric series. Halsted Press (Ellis Horwood limited, Chichester, Brisbane and Toronto.

[12]. Appell, P. and Kampé' de Feriét, J. (1926). Fonctions Hypergeometricques et Hypersperiques Polynomes d, Hermite. Gauthiervillars, paris. 